Dan Edelstein, The Terror of Natural Right. Republicanism, the Cult of Nature \& the French Revolution

Chicago, The University of Chicago Press, 2009, 337 p., ISBN

0-226-18438-2, 40 \$

Jean-Clément Martin

\title{
OpenEdition
}

Journals

Édition électronique

URL : https://journals.openedition.org/ahrf/11828

DOI : $10.4000 /$ ahrf. 11828

ISSN : 1952-403X

Éditeur :

Armand Colin, Société des études robespierristes

Édition imprimée

Date de publication : 1 juin 2010

Pagination : 259-261

ISBN : 978-2-200-92632-8

ISSN : 0003-4436

Référence électronique

Jean-Clément Martin, « Dan Edelstein, The Terror of Natural Right. Republicanism, the Cult of Nature \& the French Revolution », Annales historiques de la Révolution française [En ligne], 360 | avril-juin 2010, mis en ligne le 01 juin 2013, consulté le 24 avril 2022. URL : http://journals.openedition.org/ahrf/11828 ; DOI : https://doi.org/10.4000/ahrf.11828

Ce document a été généré automatiquement le 24 avril 2022

Tous droits réservés 


\section{Dan Edelstein, The Terror of Natural Right. Republicanism, the Cult of Nature \& the French Revolution}

Chicago, The University of Chicago Press, 2009, 337 p., ISBN

0-226-18438-2, 40 \$

Jean-Clément Martin

\section{RÉFÉRENCE}

Dan Edelstein, The Terror of Natural Right. Republicanism, the Cult of Nature \& the French Revolution, Chicago, The University of Chicago Press, 2009, 337 p., ISBN 0-226-18438-2, 40 \$

1 L'historiographie américaine relit-elle la Révolution française à la lumière des guerres menées aujourd'hui par les États-Unis contre les terroristes et autres «ennemis de l'humanité » pour reprendre une formule du président Bush? C'était l'horizon de la réflexion de $\mathrm{D}$. A. Bell à propos de la Guerre totale, et c'est la conclusion de ce livre qui, après avoir étudié la Terreur du droit naturel, critique G. W. Bush pour avoir introduit des lois d'exception ouvrant sur des perspectives mal contrôlées et rappelle que la guerre doit se mener, comme Robespierre l'a dit, la loi à la main. La démonstration est inscrite dans l'histoire des idées politiques à la suite des travaux de K. Baker, accordant une large place à l'histoire politique, voire politicienne, de la période révolutionnaire. Il s'agit d'une généalogie culturelle et de ses modalités d'application en utilisant un cas précis, la Révolution française.

Dans la première partie, l'auteur part de l'hétérogénéité des courants républicains au $\mathrm{xVIII}^{\mathrm{e}}$ siècle pour insister sur la liaison qui se produit, via la littérature, entre théories du droit naturel et républicanisme au moment où les sociétés primitives servent de modèles politiques pour rénover les sociétés occidentales. Dans ce cadre de réflexion, les individus opposés aux lois naturelles peuvent être considérés comme des ennemis 
de l'humanité. Ainsi les tyrans, les pirates, voire les habitants des nouveaux mondes auront été considérés comme tels à un moment, avant que cette lecture ne s'applique à la période révolutionnaire notamment après 1792. Dans cette évolution généalogique complexe, l'auteur s'attache à voir ce qui relie les physiocrates et S. Maréchal, par exemple, dans leurs problématiques fondées sur les lois naturelles et l'âge d'or. La Révolution est marquée par une succession de paradoxes, à commencer par les débats autour de l'abolition de la peine de mort. Les députés qui la refusent en sont, pour une part, des victimes ensuite, alors que Robespierre, qui en est partisan, est promoteur des lois de Prairial. Mais c'est la mort du roi qui représente le cas le plus important de la deuxième partie du livre. Le droit naturel permet de passer outre l'inviolabilité du roi, et de placer ce dernier hors de l'humanité. Le décret du 19 mars à l'encontre des contre-révolutionnaires s'inscrit dans cette perspective, mais l'auteur prolonge sa réflexion en lisant de la même façon la suspension de la Constitution, l'établissement $\mathrm{du}$ gouvernement révolutionnaire, les mesures de justice politique et sociale du printemps 1794 et enfin la fête de l'Être suprême et les lois de Prairial. L'horizon du droit naturel avec ce qu'il implique de refus de la volonté générale ou de toute constitution, justifie cette recherche d'un âge d'or, solaire, qui aurait été l'aboutissement de la pensée des Montagnards autour de Robespierre, de Couthon et de Saint-Just. Leur succès, temporaire, au printemps 1794 serait à lire comme la suite d'un processus enclenché depuis les premières années de la Révolution. Leur échec, en Thermidor, tient à la distance prise progressivement par les conventionnels qui récusent les conséquences de cette logique.

3 L'apport essentiel du livre tient à l'usage herméneutique du droit naturel pour comprendre le sens ultime de ce qu'a pu être la Terreur. La démarche se revendique contre-factuelle pour une part, l'auteur souhaitant mettre en valeur une logique sousjacente, en reconnaissant qu'il ne prend pas en compte la globalité et la complexité des situations, qu'il ne néglige pas pour autant. La force de la démonstration vient de cette volonté de mettre en valeur les lignes de force, sans vouloir rabattre la politique de Saint-Just ou de Robespierre sur des pratiques opportunistes, des succès occasionnels ou des ajustements. L'unité doctrinale est réelle, il n'y a pas à chercher des abandons ou des calculs, mais à saisir comment une pensée s'exprime au gré des circonstances. Dans cette lecture compliquée, la Terreur finit par être le temps de la violence la plus immédiate, acceptée faute de pouvoir faire autrement dans ce cadre annihilant les ennemis de l'humanité, avant d'être elle-même récusée au nom d'une revendication de justice naturelle, au nom d'un peuple enfin régénéré. Confirmant le passage de la Terreur à la Justice, les pages consacrées aux tentatives du printemps 1794 pour organiser le pays autour de principes issus du droit naturel, en mêlant les ambitions de Billaud-Varenne et de Saint-Just sont éclairantes et judicieuses. Ce n'est pas un hasard notamment si des tentatives de rassemblement de lois «révolutionnaires» se produisent à ce moment. Le coup de force des thermidoriens sera d'amalgamer les revendications du droit naturel, les volontés de vengeance populaire, avec cette perspective quasi eschatologique, pour rejeter la totalité de l'entreprise. La faillite du droit naturel ne tient pas à l'abandon de ses principes, mais à l'incapacité de les appliquer autrement qu'en récusant des pratiques politiciennes ou des violences incontrôlées, créant une situation politique fragile.

4 L'introduction de la dimension culturelle du droit naturel sert ici de révélateur des champs de force qui ont façonné la période. Une telle entreprise ne peut pas laisser indifférent, et soulève des questions et des objections. Il faut attendre, logiquement, la 
fin du livre pour comprendre le sens que l'on peut attribuer au mot "terreur" renvoyant à des réalités mêlées et incertaines. Les usages qui en sont faits au cours du livre posent cependant problème, hésitant entre violences, organisation politique, pratiques judiciaires ou dénonciation. Cette hésitation se retrouve dans l'emploi de formules traduisant parfois une sidération devant la violence, comme p. 132, ce chemin qui lie la Conciergerie à la place de la Révolution, alors que les forts pourcentages d'acquittement du Tribunal révolutionnaire sont cités ailleurs. Le refus exprimé de la dimension esthétique de la Terreur, dans le cadre de la réflexion sur le sublime, est discutable et rend plus difficile la compréhension des positions de Robespierre en février 1794. Les articulations entre les formes du droit naturel ne manqueront pas d'être discutées, relevons que le droit naturel chrétien est certainement trop rapidement éliminé de la discussion, alors qu'il a joué dans les prises de position des membres du Cercle social. Les liens entre républicanismes et droit naturel seront aussi discutés, notamment autour de la personnalité de Machiavel, régulièrement cité, mais sans doute pas assez discutée ni commentée. Sa réflexion sur la « fortune », entendons l'occasion saisie opportunément, aurait mérité plus d'attention, puisqu'elle aurait justifié la réussite de ce droit naturel à un moment précis de la Révolution.

5 L'examen des faits est évidemment complexe dans une pareille entreprise utilisant une érudition considérable dans une perspective d'histoire des idées qui recourt, logiquement, à des idéal-types. Les citations sont parfois biaisées. La volonté de Danton, le 27 mars 1793, que la Révolution soit peuple, dénonce dans une formule ambiguë "cette contre-révolution ». La phrase de Louis XVI, c'est légal parce que je le veux, n'est pas la pierre de touche de l'absolutisme. La ligne démonstratrice réduit la focale. Si bien qu'il manque une réflexion sur les justices prévôtales des dernières années de la monarchie absolue étudiées par T. Margadant annonciatrices des mesures de «terreur » des années 1792-1794. Enfin, Rousseau avait bien envisagé l'exclusion du genre humain des rois et des philosophes dans L'Émile, ce qui complique l'analyse. Que le décret du 19 mars 1793 n'ait pas été pris contre les Vendéens mais contre les Bretons dans un débat politique violent à la Convention modifie le point de vue, puisque, comme F. Gauthier l'a fait remarquer, ce décret suit celui pris la veille contre les partisans de la loi agraire. Tout ceci ne contredit pas Dan Edelstein mais le nuance, amenant à insister sur les inflexions provoquées par les luttes et les moments. L'unité donnée au propos empêche de prendre en compte autant qu'ils auraient dû l'être les apports d'Éric de Mari, remontant vers d'autres traditions, et d'Anne Simonin, inscrivant la Terreur dans une perspective philosophique différente.

C'est indiscutablement le lot ordinaire des ouvrages qui entendent nouer des dimensions venues de différents horizons que d'être confrontés à des attentes spécifiques et à des critiques précises. Cependant, la problématique du livre, la force de la démonstration, la subtilité des distinctions efficaces font de cet ouvrage un élément important pour résoudre les paradoxes que l'on continue de voir autour de la Terreur et pour comprendre comment les hommes du XVIII ${ }^{e}$ siècle ont confronté et modifié leurs cadres de pensée. En cela, l'auteur peut légitimement conclure sur les guerres menées contre les « ennemis de l'humanité » d'aujourd'hui, qui elles aussi s'inscrivent dans une mutation culturelle nécessaire à comprendre. 